\title{
El coaching y la transposición sociocultural como herramientas de acción pedagógica para la formación profesional
}

\section{Coaching and sociocultural transposition as tools of pedagogical action for professional training}

\author{
*Dionisio Vitalio Ponce Ruiz ${ }^{1}$, Alba Rosa Pupo Kairuz², \\ Sergio Sixto González Rodríguez ${ }^{3}$, Rosa Alba Pupo Kairuz ${ }^{4}$ \\ ${ }^{1}$ Universidad Regional Autónoma de los Andes, Facultad de Sistemas Mercantiles, Escuela de Sistemas. \\ Km 1 vía a Valencia.Quevedo,Ecuador.*Manzanillo1962@gmail.com \\ ${ }^{2}$ Universidad Técnica Estatal de Quevedo. Facultad de Ciencias Ambientales, Escuela de Ecoturismo. \\ Campus Ing. Manuel Haz Alvares, km 1.5 vía Santo Domingo de Los Tsáchilas. EC.120501. Quevedo, \\ Ecuador.apupo@uteq.edu.ec \\ ${ }^{3}$ Universidad Regional Autónoma de los Andes, Facultad de Administración, Escuela de Administración. \\ Km 1 vía a Valencia. Quevedo, Ecuador. gsergiosixto@yahoo.es \\ ${ }^{4}$ Investigadora independiente, Bayamo, Granma,Cuba. rpupo@gmail.com
}

Rec.: 25.02.2016. Acept.: 12.05.2016.

Publicado el 30 de diciembre de 2016

Rev. Cienc. Soc. y Econ. UTEQ (2016), 1(1): 23-41

\begin{abstract}
Resumen
El presente trabajo parte de la problemática real que hoy aparece en el ámbito educativo mundial: ¿contribuyen las ciencias pedagógicas a la formación pertinente de los sujetos ante los desafíos postmodernos? el objetivo se centra en presentar una reflexión teórica, sobre las relaciones entre coaching, transposición y pedagogía, con el fin de socializar entre los docentes universitarios nuevas formas pedagógicas comunicacionales para transformar las realidades educativas en las universidades. Se ha considerado importante emplear, el método dialéctico, la teoría de la complejidad y la teoría general de sistemas, para profundizar en este debate y presentar una propuesta transformadora de la problemática abordada. Se presenta como resultado la modelación de una propuesta pedagógica susceptible de ser contextualizada.
\end{abstract}


Palabras clave: coaching, diálogo cultural, transformación humana, procesos conscientes, formación universitaria.

\begin{abstract}
This paper departs from a real problematic emerging in the world educative scope today: do pedagogical sciences contribute to the pertinent formation of the subjects in face of postmodern challenges? It aims to present a deep theoretical reflection on the relationships between coaching, transposition and pedagogy, in order to socialize among university staff new pedagogical, communicational ways to transform educational realities in universities. We have considered important to use dialectical method, the theory of complexity and the general theory of systems, to deepen this debate and to present a transformative proposal of the problem addressed. The model of a pedagogical proposal susceptible to be contextualized is presented.
\end{abstract}

Keywords: coaching, cultural dialogue, human transformation, conscious processes, university education.

\title{
Introducción
}

La llamada "sociedad del conocimiento" y la presencia de múltiples "ismos"; que pretenden explicar la realidad social y cultural del mundo en el siglo xxi, hacen necesaria una reflexión pedagógica profunda. Tiene sentido que la pedagogía, en tiempos de postmodernidad -dada su importancia para afrontar los cambios sociales, políticos y tecnológicos comunicacionales ocurridos-, deba replantearse sus presupuestos, principios, y prácticas (Didrikssom, 2009).

Una pedagogía universitaria, pertinente frente al entorno, las tensiones y las contradicciones que hoy determina el desarrollo de los seres humanos; debe si quiere, ser realmente posmoderna, abordar las actitudes, representaciones y deseos de las nuevas generaciones, nuevas formas de identidad y cartografías culturales han condicionado la generación de representaciones sociales nuevas (Álvarez, 2000), las cuales deben entenderse en el contexto de prácticas culturales novedosas e híbridas, es necesario que los educadores entiendan cómo están surgiendo diferentes identidades, en medio de la 
sociedad globalizada, en ámbitos y esferas de actuación que las escuelas generalmente obvian la pedagogía debe redefinir su relación con estas nuevas formas de la cultura; como práctica cultural crítica necesita abrirse a nuevos espacios institucionales en donde los estudiantes puedan experimentar y definir el significado de ser gestores axiológicos culturales (Bustelo, 1993).

Ahondar en el coaching, y la trasposición sociocultural, es un desafío al cual se desea asistir hace mucho tiempo, en honor a la verdad, este es aún un terreno en construcción, todavía no existen definiciones acabadas, pues el decursar histórico ha condicionado miradas dialécticas diversas a estos aspectos de la realidad comunicacional y educativa (Ponce, 2011). El abordar estas categorías implica la elaboración de propuestas pedagógicas reales para nuestras aulas, ello convoca a apuntalar en esta batalla. Por consiguiente, el objetivo de este trabajo es valorar los conocimientos básicos que poseen los profesionales acerca de su concepción de la gestión axiológica cultural en el desempeño profesional.

\section{Materiales y métodos}

La investigación se desarrolla en el ámbito cualitativo a partir de considerar los procesos de formación de los profesionales, en sus contexto culturales profesionales y genera mediante la teoría fundamentada, un modelo de gestión de la formación, junto al método de la transposición sociocultural. Se apunta también un análisis de otras perspectivas pedagógicas como el coaching ontológico.

Como métodos de investigación se utilizaron en el orden teórico: analítico -sintético, inductivo- deductivo, histórico-lógico, la modelación y el sistémico-estructural. Se realizaron entrevista, encuestas y talleres con gestores culturales.

\section{Resultados y discusión}

El coaching aproximación filosófica y epistemológica, desde el ser humano y la condición humana 
Los grados de desorientación que actualmente presentan los seres humanos, expresan la realidad de una profunda crisis existencia, que nos lleva a no construir significados y sentidos en nuestras vidas. Hay dos elementos claves en este análisis y pasan por las categorías sufrimientos e incapacidad. Ello nos lleva a sensaciones de impotencia que van cercenando una forma de comportamiento en la cual los seres humanos se enajenan se entregan a la espectacularidad contemporánea como salida a sus necesidades de autorrealización espiritual.

La mirada del profesor cubano, Fuentes (2010) sobre estos temas y su propuesta de la construcción de una universidad humana y cultural (Figura 1), colocan ante una interpretación de su modelo de la condición humana.

\section{REPRESENTACIÓN DE LA CONDICIÓN HUMANA}

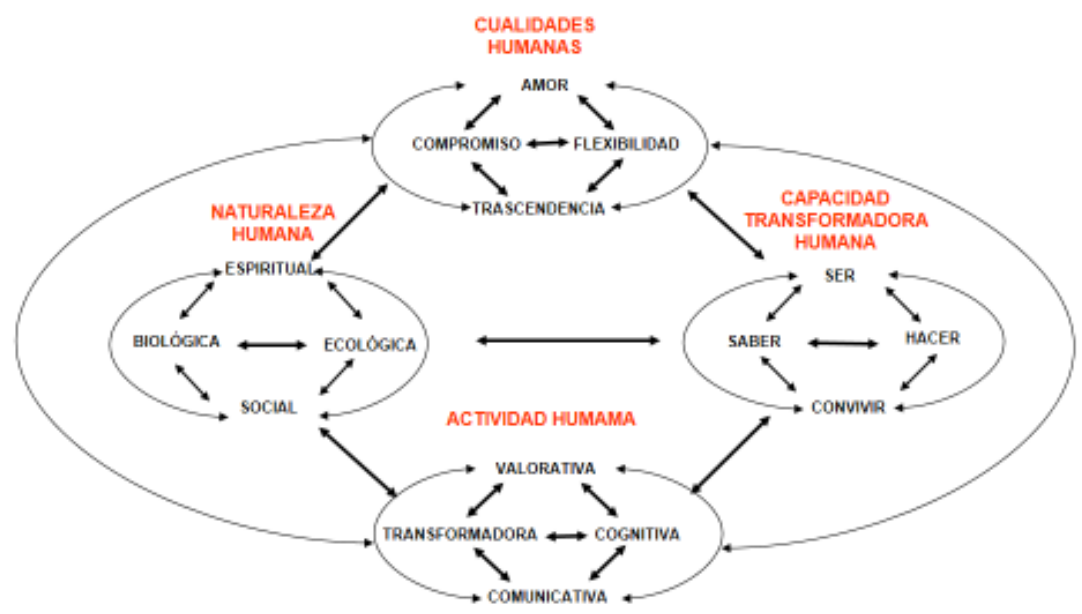

Figura 1. La condición humana. Tomada de conferencia del Dr. Fuentes (2010)

Aquí es importante resaltar el carácter relacional y holísticos del desarrollo humano y como la actividad humana es transcendental en los procesos de formación.

\section{La ontología del lenguaje y el coaching ontológico}

Al entender que el lenguaje posibilita forjar futuros diferentes, asumiendo que los procesos conversacionales, radican las potencialidades pedagógicas del coaching, conduce a una reflexión sucinta sobre el largo camino de este para su aparición, en esto tiene preponderancia las opiniones del profesor Rafael Echevarría, desde el cual hemos podido asumir y trasmitir algunos referentes históricos que ayudan a entender este hecho. Al pensar que el lenguaje es acción, se abre para la filosofía una nueva manera de entender 
las relaciones humanas. En este punto del análisis, aparecen dos dimensiones que son importantes, la noción de los actos del lenguaje, aquí aparecen las acciones, y esto nos remite a la acción comunicativa de Jürgen Habermas, al propio tiempo aparecen competencias en los seres humanos, lo cual abre en un campo cultural trascendental, para conformar el ser humano, sus rasgos y sus identidades.

La discusión sobre estos temas nace en la modernidad, cuando resurge la pregunta por la condición humana, esto se potencia a partir de Kant y su interrogante ¿qué es el hombre? en esta época, el pensador Friedrich Nietzsche cuestiona la tradición de pensamiento occidental y señala la posibilidad de una nueva mirada al hombre, revela la crisis en el ámbito de la ética, en el mundo de los valores, haciendo una referencia al nihilismo, que aún perdura él es el primer pensador que apunta a Heráclito, y afirma que el mundo está en permanente transformación y se puede vivir en ella, para él la acción es esencial y el lenguaje tiene una importancia capital.

Otro referente importante según Echevarría (2014), para entender el surgimiento del coaching es Martin Heidegger, quien da respuesta a como somos, al afirmar que el lenguaje es la morada del ser. Esta idea es potenciada por Martin Burger, quien abre espacios al ser dialógico y establece tres ejes conversacionales, trascendentales en la construcción de significados y sentidos, a saber:

- de la conversación con los otros.

- de la conversación con nosotros

- de la conversación con el misterio de la vida, es decir con dios y la eternidad.

Desde estas ideas preliminares, se precisa revisar el giro lingüístico, al colocar al lenguaje en un plano de interpretación sobre el lenguaje todavía no aceptados plenamente, aquí impactan Ferdinand de Saussure, la aparición de la hermenéutica y su relación con los textos y contexto, el desarrollo de la antropología. Los principales aportes surgen desde la filosofía del lenguaje, con las obras de Ludwig Wittgenstein al asegurar que todo lenguaje es una forma de vida, quien ve al lenguaje como una forma activa de la acción, por lo cual es transformador al generar lenguajes distintos.

Estos desarrollos coinciden con los planteamientos de Freire (1999), McLaren, (2000), Maturana \& Varela (2002), alcanzando su pináculo epistémico en lo que hoy se llama coaching ontológico, promulgado por Echevarría (2015), el cual propone la ontología del lenguaje y el coaching ontológico (Figura 2). Los cuales parecen muy interesantes 
para el desarrollo de la cultura dialógica en los seres humanos. Por ello se presenta su modelo osar, como modelación importante para la educación.

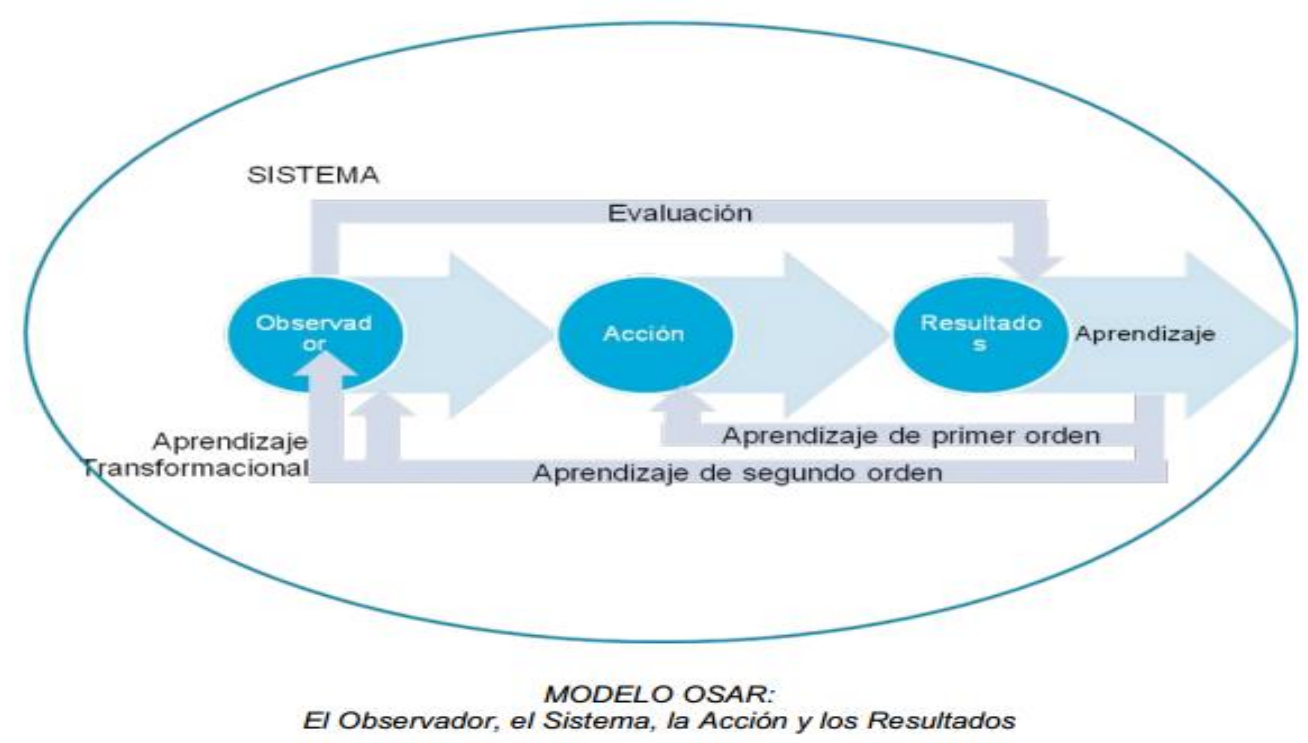

Figura 2. Modelo osar. Tomada de conferencia del Dr. Echeverría (2015)

Los aportes de este modelo, se constituyen en oportunidades para introducir en los procesos pedagógicos, investigativos y de vinculación en las universidades como posibilidad de edificación de una nueva forma de construcción de la relación docenteestudiante-contenido profesional. Si los educadores entendieran el valor de las conversaciones, cambiarían los procesos de orientación, evaluación y tutorías, con resultados más trascendentales en la formación profesional.

\section{La transposición sociocultural, análisis desde lo histórico-conceptual en al ámbito} de la sociología y la educación

La historia de las categorías culturales que aluden al dialogo y la construcción de referentes identitarios, remiten a la obra de Ortiz (1962), Malinoswki (1985), García (2005), Maffessoli (2001), Franzone (2007) entre los más destacados investigadores en esta materia. En este aspecto resaltan las siguientes: aculturación, inculturación, transculturación, mestizaje e hibridación cultural.

Cada una con sus resonancias y ontologías interpretativas propias sobre el fenómeno de intercambio cultural, así como las huellas en el aprendizaje y la identidad de los pueblos. 


\section{La transposición cultural, potencialidades pedagógicas}

Dada la importancia que se realiza a este constructo se hace necesario en primer lugar situar diversas acepciones, que permitan entender que la transposición es reconocida como:

- Figura retórica de construcción que consiste en la alteración del orden sintáctico que se considera habitual y lógico de las palabras de una oración.

- Cambiar de lugar a una persona o cosa

- Ir de un lado al otro de una cosa

Aquí se expresa el sentido de trasladar y cambio, que es ontológicamente reconocido los procesos de transposición.

\section{Reflexiones sobre los orígenes y la actualidad formación de los profesionales como gestores axiológicos culturales.}

Fijar las coordenadas epistemológicas de la relación formación-cultura precisa ahondar en dos definiciones sobre la categoría cultura. en primer lugar la asumida Hart (2001), presidente de la sociedad cultural José Martí, desde de la siguiente interrogante, que él mismo dilucida: “...¿qué es la cultura? la singularidad humana en la historia natural radica en el hecho de que el hombre, al tomar conciencia de su propia existencia, de su pertenencia a la naturaleza, se planteó como exigencia descubrir y descifrar el misterio de lo desconocido. Los hombres, por su carácter de entes biopsicosociales, son los únicos seres vivientes que tienen ese reto, de ahí nace la cultura hasta convertirse en segunda naturaleza, ella es, a la vez, claustro materno y creación de la humanidad no hay hombre, en el sentido pleno y universal del término, sin cultura y esta no existe sin aquél su afán de descubrir lo que no conoce lo lleva al extremo de intentar encontrar el sentido de su propia existencia."

Esta consideración devela la existencia, mediada por la relación hombre-cultura, de un proceso de apropiación sociocultural en el sujeto que dinamiza el sentido de la existencia humana y condiciona su interpretación de la relación entre la cultura, la formación y los contextos culturales, es importante considerar, además, que en esa definición se expresa 
el carácter de indagación permanente que el sujeto social encuentra en sus relaciones culturales.

En segundo lugar se considera la definición de cultura aportada por Montoya (2005), quien revela su valor al plantear que es un proceso íntegro y dinámico de productos supra naturales e intersubjetivos relacionados dialécticamente, devenidos de las actividades objetiva y subjetiva del hombre, expresados y extendidos como resultados acumulados, creaciones constantes, proyectos y fines, para satisfacer las necesidades del sujeto social, en un periodo históricamente determinado de su realidad contextual, que inciden de manera directa en el proceso de preparación, formación y desarrollo de la personalidad en su socialización e individualización y condicionan el avance y progreso de la sociedad humana:

Al interpretar teóricamente esta definición se puede comprender la importancia de la cultura como expresión directa de la actividad humana y su trascendencia en la educación del hombre. Esta definición permite abordar los procesos de socialización de los cuales es partícipe el sujeto en los contextos culturales y por otra parte revela dinámicas que se pueden constituir en concreciones específicas en la actividad educativa como vehículo de humanización.

Las valoraciones realizadas dan una aproximación al concepto de cultura: como expresión histórico-concreta de la interrelación hombre-naturaleza, que permite la observación, abstracción y concreción práctica del sujeto social en los contextos socioculturales. Ella se erige en capital intelectual de la humanidad y mecanismo a través del cual los seres humanos crean y recrean los significados y sentidos compartidos, como resultado de la práctica social y los procesos comunicativos que en ésta se da (Ponce, 2011).

La deconstrucción de esta idea permite el reconocimiento de las relaciones de la cultura con el proceso de formación de los profesionales en la educación superior, al considerar el contexto sociocultural, teniendo en cuenta que en la actualidad los entornos socioculturales están muy presentes en la conformación de prácticas humanas de socialización.

Si se comparte el criterio de que la cultura es resultado de la práctica social del hombre en su relación con la naturaleza y fruto de la historia de la humanidad, se está enfatizando entonces en el valor social de ésta, lo que le confiere potencialidades al medio sociocultural en la formación de los sujetos. En este sentido, es importante considerar que resulta insuficiente la 
comprensión que se tiene en las universidades del carácter polivalente de la cultura contextual y siendo limitada su sistematización durante el proceso de formación cultural profesional.

Sí de modo general, se puede considerar la cultura como un sistema de hábitos y costumbres propios de los seres humanos, adquiridos a través de sus procesos de participación social y empleados como su principal mecanismo de adaptación y transformación del medio o contexto donde desarrollan su vida; aunque no estén todavía totalmente dilucidadas las relaciones y nexos que se dan en este proceso, la idea permite comprender la importancia de considerar la cultura y la diversidad sociocultural como factores influyentes en los espacios de formación de los profesionales en la educación superior.

Ello condiciona la existencia en la formación de los estudiantes universitarios de procesos conscientes de apropiación de dicha diversidad, los que se convierten en mecanismo de autoformación sociocultural de los mismos. Al profundizar en las esencialidades de estos procesos de formación, es importante apuntar que Fuentes (2010) los concibe como proceso y fin en la educación superior, al precisar que la formación de los profesionales debe tener un carácter consciente al desarrollarse en el espacio de construcción de significados y sentidos por los sujetos que intervienen, lo que le evidencia su enfoque holístico y dialéctico.

El mismo autor reconoce que el proceso de formación de los profesionales está determinado por la necesidad de formar a las nuevas generaciones y de educar, en general, a la población; de ahí la naturaleza social del proceso por el carácter consciente de los sujetos, cuyo objetivo es su propia formación para su desempeño social y profesional, siendo el contenido la cultura social y profesional apropiada por los sujetos en términos de conocimientos, habilidades, valores y valoraciones, los que se expresan en sus competencias profesionales y sociales.

Se ha constatado que algunos autores han denotado la relación cultura-formación a partir de variadas posturas indagatorias al respecto, la relación entre la cultura y la educación de forma general y en particular la formación profesional, ha sido abordada por Álvarez (1999), Olmos (2002), Addine (2004), Basail et al. (2005), Fernández y Parajeles (2005), Montoya (2005), Basora (2007), Espinosa (2008), Fuentes (2008), entre otros.

Estos autores reconocen la cultura como contenido para la educación del hombre y conceden importancia a la relación sujeto-contexto, sin embargo, aún es insuficiente el tratamiento epistemológico de dicha relación, en función de determinar las posibilidades participativas y activas del hombre en su propia formación en una dinámica de interrelación cultura académica-cultura contextual. Al no ser tratada esta interrelación con regularidad, no se han 
dilucidado todas las aportaciones que los contextos socioculturales y sus identidades puedan dar a la formación profesional del sujeto.

Por ello se precisa una aproximación a la cultura en su dimensión educativa, lo cual permitirá abordar elementos esenciales en la construcción científica que se pretende. Se debe consignar que en las indagaciones realizadas por Álvarez (1998), Álvarez (2000), Álvarez (2001), Zilberstein (2002), Montoya (2005), Horruitiner (2007), Fuentes (2008), Didrikssom (2009), Espinosa (2009) y Fuentes (2010) entre otros, se destaca la formación del profesional como proceso, el papel de la cultura como contenido y la determinación social de la educación; en un proceso dirigido a la preservación, desarrollo, difusión y creación de la cultura.

En estos autores se encuentran referentes valiosos; aunque se hace necesario profundizar en el estudio de las potencialidades de la cultura contextual para la formación del hombre, lo cual condiciona una aproximación necesaria a los procesos de identidad cultural, memoria colectiva e imaginarios sociales, categorías no tratadas plenamente en el contexto de la formación de los profesionales como gestores socioculturales, a pesar de toda la intencionalidad y riqueza que ellas aportan a la educación.

En el análisis del proceso de formación de los profesionales como gestores culturales se evidencian varios elementos a tener en cuenta. Un primer aspecto es el relativo al origen y la definición de la gestión cultural. lo anterior remite a las consideraciones generales sobre la gestión empresarial y a las investigaciones de Drucker (2002), el cual asevera que entre las décadas de los años 50' y los 80’ del siglo xx, las organizaciones evolucionan en un período al que llama "revolución de la gestión". Es en este tiempo donde surge el planteamiento que considera las organizaciones culturales como estructuras intensivas en complejidad (Martinell, 2001).

Para los autores de esta investigación la gestión cultural es un proceso de dirección en función de la creación y el desarrollo de la cultura. Ello cobra significación al ser una disciplina científica que toma los principios generales de la gestión, pero que se contextualiza en los procesos culturales, lo que demanda una nueva epistemología y metodología; para valorar en su campo de acción, las estructuraciones y las dinámicas del amplio espectro de actividades, eventos y procesos relacionados con el universo cultural profesional en que actúan los graduados universitarios. 
La formación de los profesionales propicia el aprendizaje socio profesional contextual el cual ha sido esencial en el desarrollo de la sociedad. El mismo se da en la actividad y la comunicación, lo cual acontece en un contexto sociocultural determinado y se propicia a partir del diálogo cultural. El tema, ha sido pretensión de numerosas escuelas y de destacados pensadores como Maffesoli (2001), Morín (2001) y García (2005) abordan el carácter dialógico de la adquisición de los conocimientos. Estos diálogos ocurren en espacio y tiempo y son condicionados por herencias culturales y factores que establecen un entorno socio-comunicativo polivalente.

La realidad formativa universitaria, produce estos procesos de aprendizaje, pero desconoce las posturas científicas que refrendan la transposición de saberes, lo cual a juicio de esta investigación abre una posibilidad nueva en la formación del hombre.

\section{La transposición sociocultural, el modelo transpositivo y su método}

El concepto de la transposición sociocultural, como lo planteara Ponce (2011), se elabora desde consideraciones pedagógicas relativas a la transculturación y la hibridación cultural, como procesos reconocidos por las ciencias sociales. para el sostén teórico se ha penetrado en la esencialidad de estos procesos de intercambio cultural lo que permite plantear el siguiente concepto (Figura 3): “la transposición sociocultural es un complejo proceso de relaciones y transformaciones de los imaginarios socioculturales, que permite la formación de los sujetos a partir de su interrelación en los contextos culturales, en una dinámica de reconocimiento, apropiación, intervención y validación sociocultural que puede connotar los comportamientos humanos, las prácticas culturales y las cosmovisiones". 


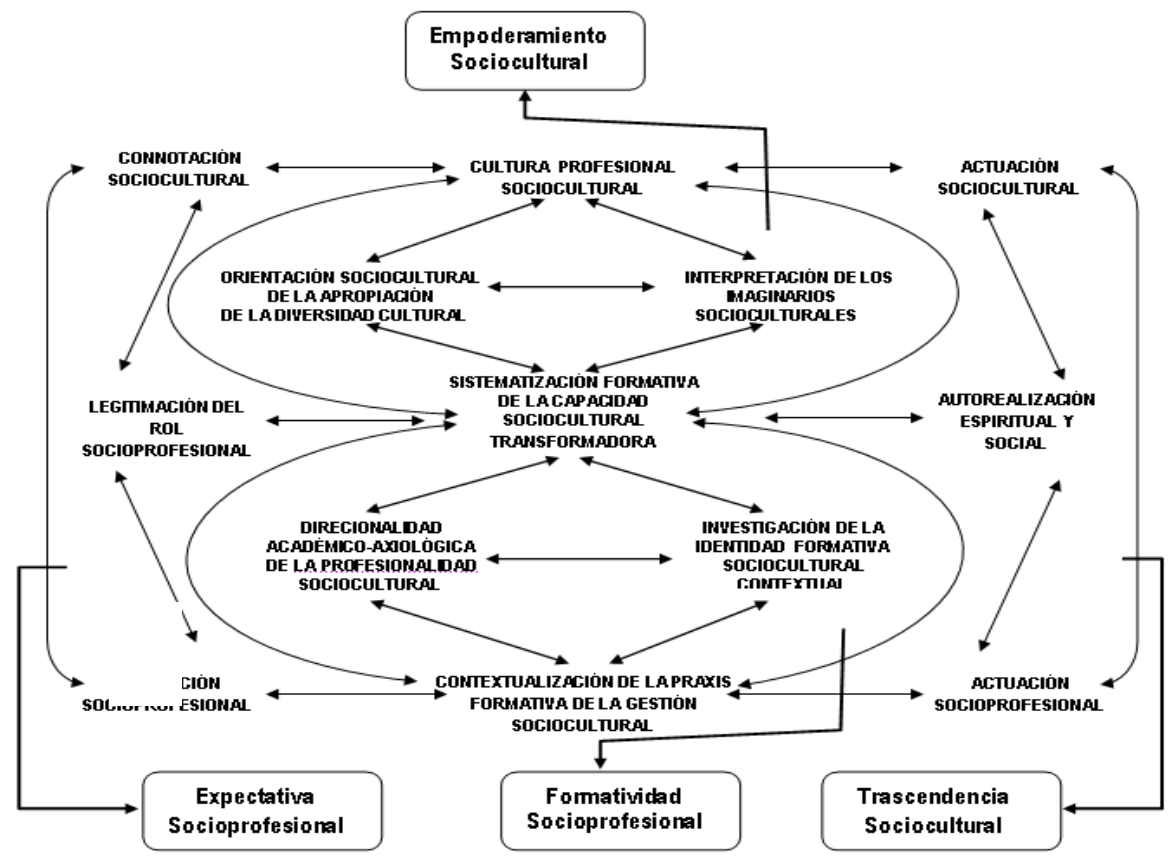

Figura 3. Representaciones del modelo de la dinámica formativa en gestión sociocultural

Este modelo condiciona la emergencia del método de la transposición sociocultural, que se estructura a través de tres procedimientos. Estos son: procedimiento de formación del reconocimiento, apropiación y validación sociocultural; procedimiento de formación de la concretización de la transposición sociocultural en contextos; procedimiento de formación del auto reconocimiento formativo sociocultural.

El aprendizaje profesional en las universidades. Los espacios formativos una mirada desde la transposición sociocultural

El proceso de formación de los profesionales como gestores culturales, a tenor del modelo propuesto posibilita estructurar formas de intervención en las dimensiones académicas, investigativas y extensionistas en nuestras universidades, permitiendo el empoderamiento de los estudiantes (Figura 4). Ello obliga a un giro en la reflexión desarrollada para evidenciar el valor en la praxis de las propuestas conceptuales y metodológicas tanto de la transposición sociocultural, como de su método. Por tanto se precisa analizar los cánones tradicionales del aprendizaje y algunos de los referentes universales en cuanto a la adquisición de conocimientos desde la cultura occidental., se destacan las ideas de Lenin (1985), Morín (2001), Habermas (2003) y Fuentes (2010) las cuales se valoran a continuación. 


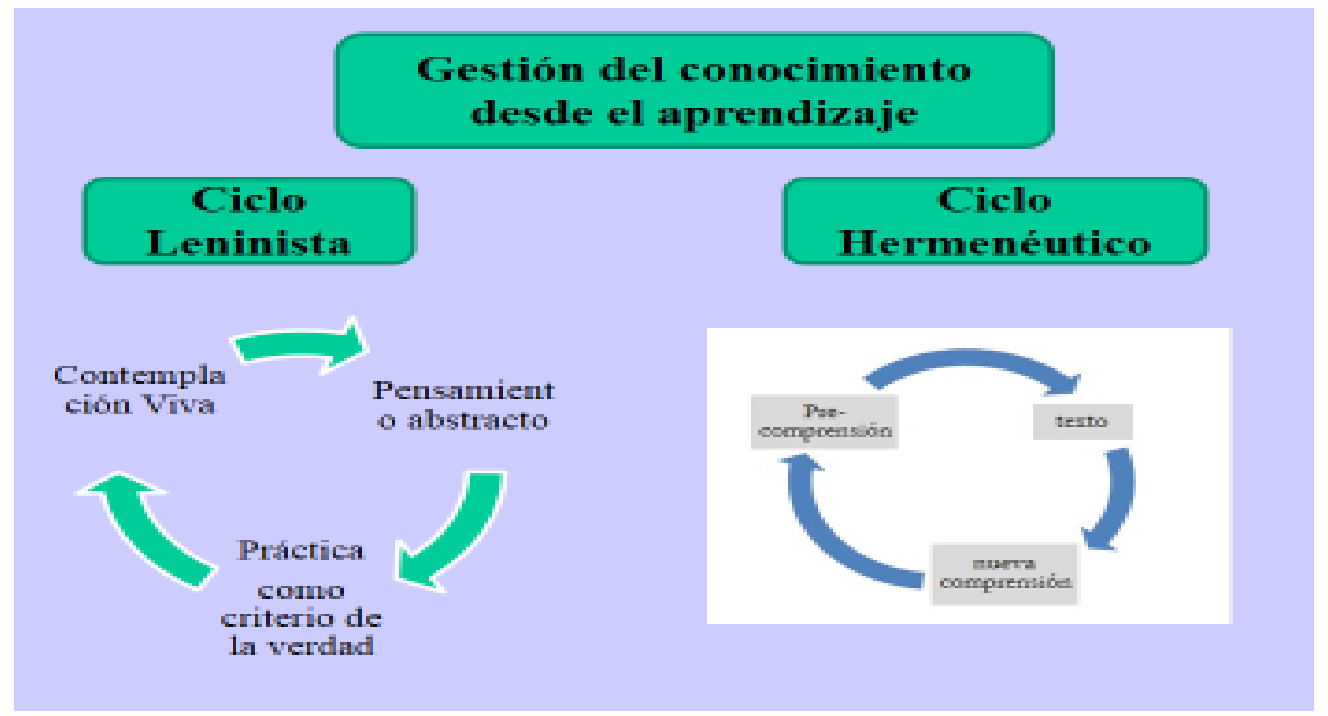

Figura 4. Ciclos del conocimiento: leninista y hermenéutico

Se destaca en este gráfico la contrastación entre ambos ciclos unos, el leninista, visto como un camino de espiral ascendente con un sentido dialectico, pero que en alguna medida recuerda o conduce al reconocimiento de la mejora continua y otro, el de Habermas, más centrado en la precondición y la condición desde la interpretación del texto y el contexto educativo, lo cual le da un sentido hermenéutico. Ambos de mucha valía para el enfoque y construcción del conocimiento, a nuestro juicio, van a depender de la maestría pedagógica y el ámbito educativo para su correcto empleo por los docentes (Figura 5).

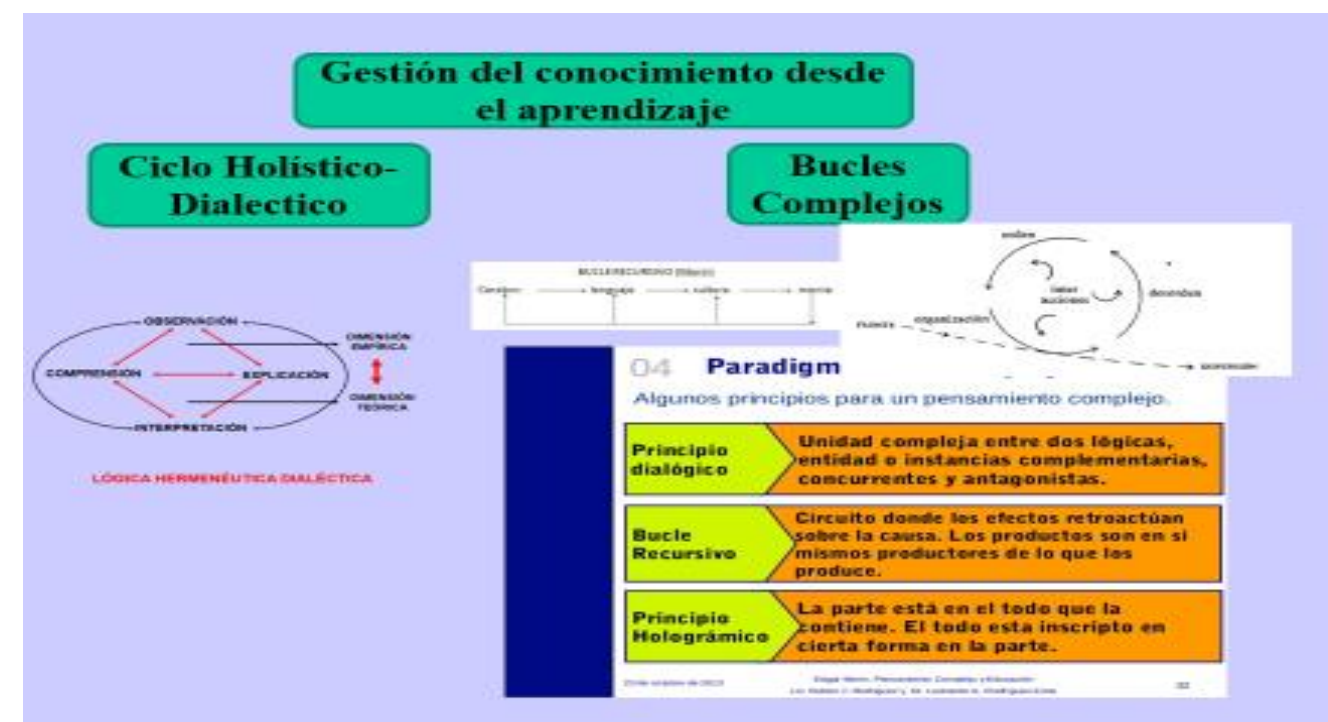

Figura 5. Ciclos del conocimiento: holísticos-dialéctico y bucles complejos 
En este caso, la figura 5, nos aproxima a dos miradas actuales de la construcción del conocimiento, las cuales consideramos importantes, por su carácter dialectico, transvivencial y transdisciplinar. En primer lugar el ciclo holístico-dialectico, del profesor Homero Fuentes, que en suma es una configuración proyectiva, enfocada en ampliar el alcance de la lógica hermenéutica dialéctica y por otra parte, la mirada de E. Morín a partir de la relación orden-desorden- reordenamiento, que le da un tránsito dialectico al fenómeno de la complejidad en el ámbito del aprendizaje.

Es en estos complejos momentos de la reflexión pedagógica didáctica sobre el aprendizaje profesional que nos parece pertinente introducir el concepto de entornos transpositivos de aprendizaje, que son los espacios de reflexión dialógica critica emprendedora que se propician al interior de los ámbitos de educativos, desde el entendimiento del dialogo como eje vertebrador de la construcción cultural del saber y el hacer humano.

Entendiendo que desde la construcción de las representaciones sociales, existen dos formas aparentes de dominio de la cognición, que son, la parte central del saber al cual llamamos núcleo y la parte periférica. Estos entornos parten de reconocer, las esencias de la transposición sociocultural y como los actores que participan de los mismos pueden establecer conexiones a partir de sus imaginarios culturales en una dialógica que se da a nivel relacional de tres modos explícitos, a saber: modo 1 relación núcleo - núcleo, modo 2 relación núcleo - periferia y modo3 relación periferia-periferia.

Estas relaciones determinaran, las acciones pedagógicas y didácticas que desde la lógica transpositiva y el método propician el aprendizaje profesional y el desarrollo integral profesional en los actores del proceso. Estudios desarrollados en este campo nos permiten aseverar que un docente sensible a su entorno cultural, con un dominio avanzado de los contenidos de su asignatura, y con una capacidad de reconocimiento sociocultural y psicológico de sus estudiantes puede generar experiencias formativas en los ámbitos docentes aplicando el método de la transposición sociocultural y desarrollando entornos transpositivos de aprendizaje.

\section{Instrumentación en el ámbito universitario. Estrategia pedagógica}

A modo de constatación de la valía del modelo y el método desarrollado se han intervenido en diversos escenarios educativos, siempre con el objetivo de potenciar la 
formación de los profesionales como gestores axiológicos culturales (Figura 6). Esta experiencia socio-formativa han sido desarrolladas en el centro universitario municipal de manzanillo, cuba en un periodo de cuatro cursos de la carrera estudios socioculturales, las experiencias obtenidas han sido generalizadas en talleres formativos, eventos científicos y seminarios en Cuba, Colombia y Ecuador.

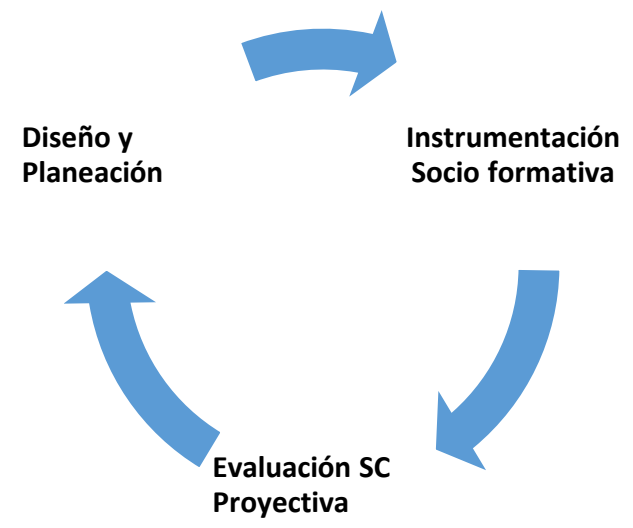

Figura 6. Sistema estratégico proyectivo

Para concretar en la práctica pedagógica, la aplicación de la transposición sociocultural, el modelo de formación de los gestores y el método de la transposición sociocultural, se gesta todo un proceso complejo y sistémico donde se establecen tres etapas para el desarrollo de la estrategia que a continuación se describen.

\section{Etapa diseño y planeación proyectiva}
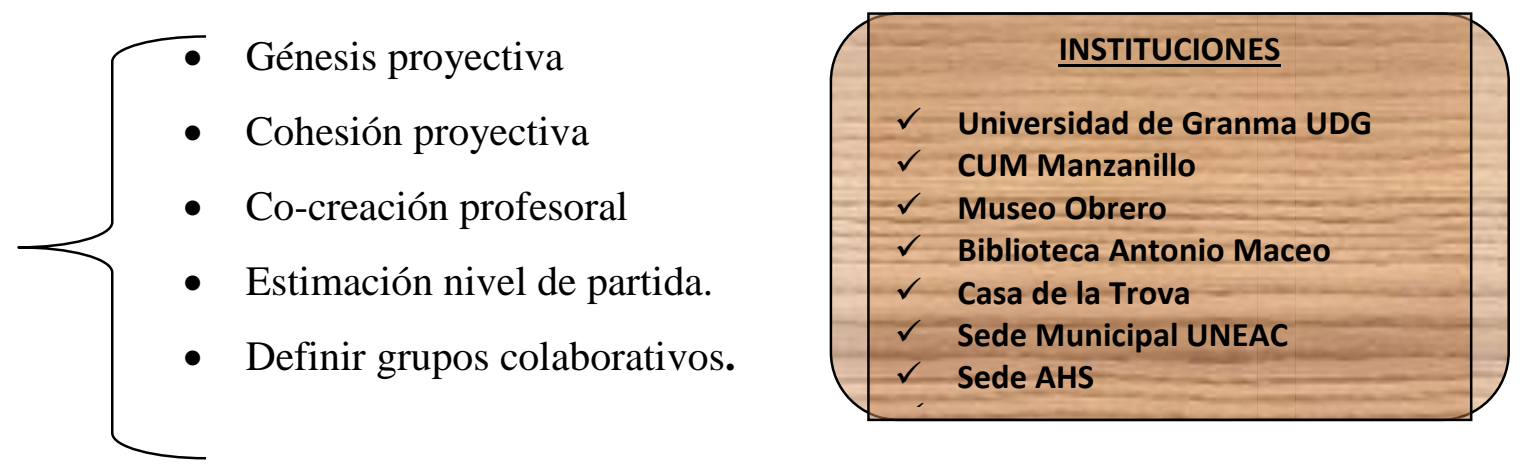

Figura 7. Diseño y planeación

Es importante entender como en esta etapa el valor primordial lo tienen dos ejes esenciales en la práctica pedagógica-didáctica, a saber: el reconocimiento sociocultural, a nivel de individuos, aulas e instituciones involucradas y el planteo de los elementos de co-creación profesoral, como respuesta al problema pedagógico investigado, así como el 
entorno de solución del mismo. Aquí vemos cómo se logra establecer una red de instituciones que por su esencialidad cultural son coherentes con la naturaleza del proceso formativo que se pretende.

\section{Etapa interventora socio-formativa}

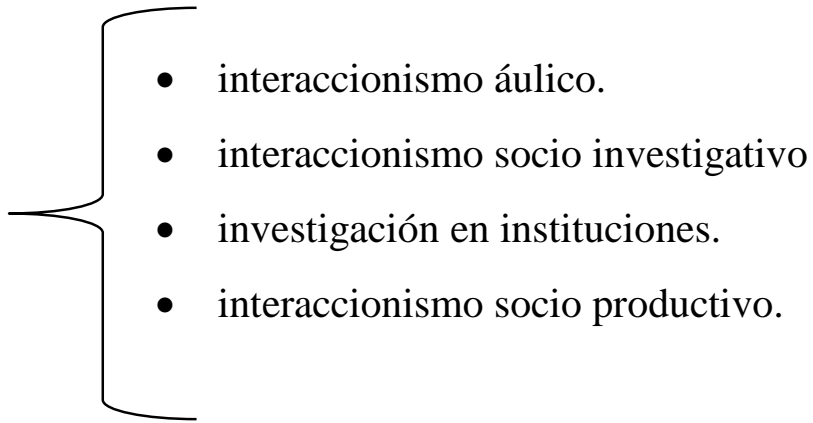

Figura 8. Etapa interventora socio-proyectiva

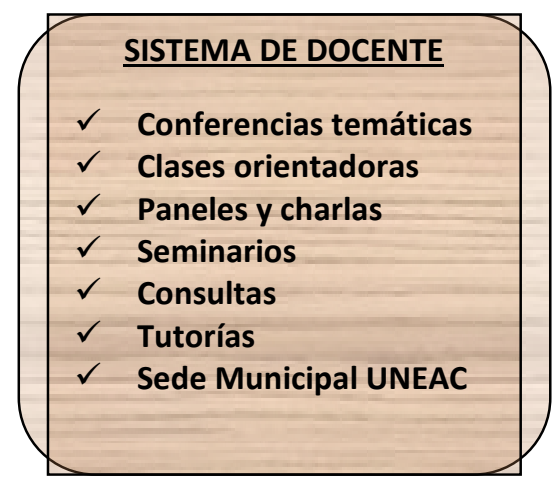

Aquí adquiere trascendencia el segundo procedimiento del método transpositivo sociocultural, al condicionar las lógicas de estructuración y la asignación de roles en los actores que participan del dialogo transpositivo y la construcción de nuevos saberes y por tanto nuevas representaciones e identidades.

\section{Etapa evaluativa sociocultural proyectiva}
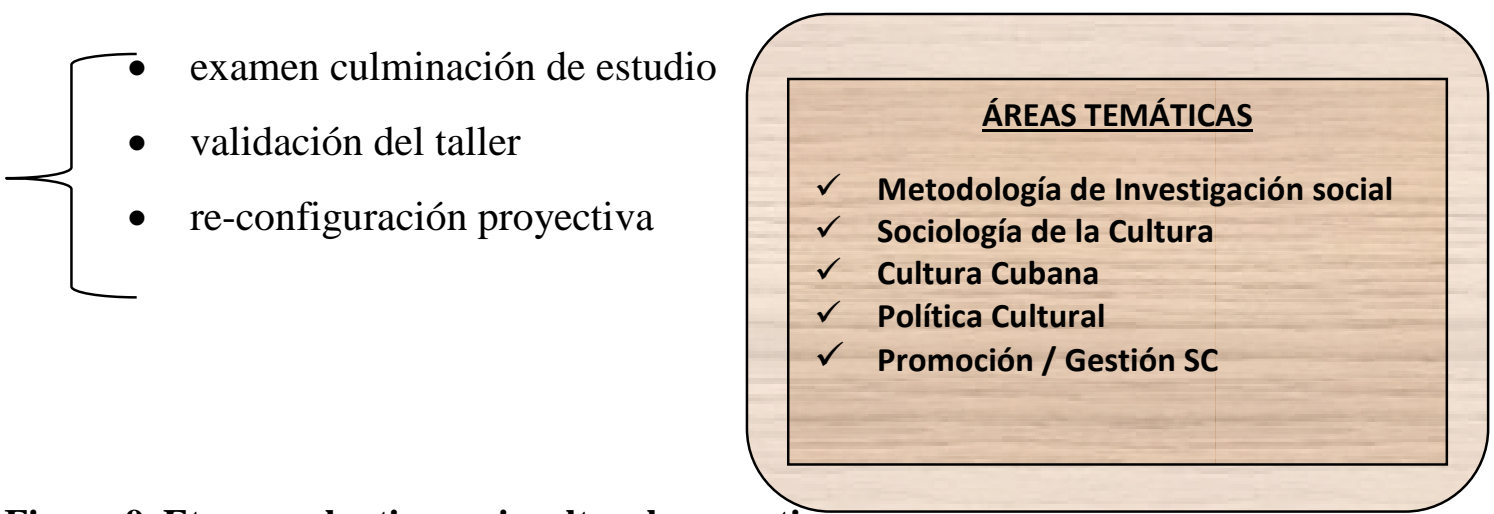

Figura 9. Etapa evaluativa sociocultural proyectiva

En esta etapa se gestó la reconstrucción de escenarios desde una perspectiva dialéctica trasformadora en búsqueda de una mejora continua, por ende prima el ámbito evaluativo de la experiencia desde el tercer procedimiento de formación del auto reconocimiento formativo sociocultural. 
Esta experiencia formativa aporto además elementos de plasticidad de la metodología, pues quedo demostrado que al interior de algunos actos didácticos se podía incorporar dinámicas de coaching, trabajo grupal y representatividad que enriquecen las apropiaciones y sistematizaciones culturales desarrolladas.

\section{Conclusiones}

La reflexión desarrollada sobre los desafíos y las nuevas tendencias en la formación universitaria de los profesionales, han conducido al reconocimiento del coaching, como una trascendental posibilidad pedagógica, en el marco de la sociedad actual.

La presentación del modelo de la dinámica formativa de la gestión sociocultural enriquece la visión científica de la formación de los profesionales como gestores axiológicos culturales a partir de la observación, comprensión, explicación e interpretación de la relación sujeto-contexto cultural; ello formaliza una propuesta intencionada para la sistematización de la capacidad sociocultural transformadora de los profesionales.

Con la elaboración del método de la transposición sociocultural se aporta un instrumento susceptible de ser usado en los procesos de formación de los gestores axiológicos culturales que considera sus carencias, al potenciar en éstos el desarrollo de su capacidad sociocultural transformadora en una perenne autoformación que tiene como base el dialogo sujeto-contexto cultural.

\section{Referencias bibliográficas}

Addine, F. (2004). Teoría y Práctica. La Habana, Editorial Pueblo y Educación.

Álvarez De Sayas, C. (1998). Fundamentos teóricos de la dirección del proceso de formación del profesional del perfil amplio. Universidad Central de Las Villas.

Basail, RA., Dávalos, DR. (2005). Antropología Social. Selección De Lecturas. Ciudad de La Habana, Félix Varela. Cuba.

Basora, O. (2007). Modelo de Gestión de la formación y de la identidad universitaria. [Tesis de Doctor en Ciencias Pedagógicas]. Santiago de Cuba. Cuba. 
Bustelo, M. (1993). La evaluación de los procesos socioculturales (Material mimeografiado).

Didrikssom, TA. (2009). Una nueva universidad para la sociedad del conocimiento. Revista Temas, 57.

Drucker, P. (2002). La gerencia en la sociedad futura. Editorial Norma. Bogotá. Colombia.

Echeverría. R. (2015). Ontología Del Lenguaje. Lom Ediciones S.A. Chile.

Espinosa, RJ. (2008) Gestión De La Cultura Profesional En La Educación Superior. [Tesis Presentada En Opción Al Grado Científico De Doctor En Ciencias Pedagógicas]. Bayamo. Cuba

Fernández, MV. \& Parajeles, SJ. (2005). La formación del profesorado en la enseñanza de los estudios sociales en la una y UCR: Un análisis sobre la perspectiva vigente y las necesidades que demanda el modelo educativo costarricense. Costa Rica.

Franzone, M. (2007) Comprender las estructuras imaginarias de Gilbert Durand. Argentina.

Freire, P. (1999). Pedagogía del oprimido. Editorial Siglo Veintiuno. México.

Fuentes, GH. (2010). La Formación De los profesionales en la educación superior. Una alternativa holística, compleja y dialéctica en la construcción del conocimiento científico. Santiago de Cuba, CEES Manuel F. Gran. Cuba.

Fuentes, L. (2008). El Método Etnográfico En La Formación De Profesionales. [Tesis Presentada En Opción Al Grado Científico De Doctor En Ciencias Pedagógicas].Cuba

García, CN. (2005). Todos Tienen Cultura. García, Conferencia para el Seminario sobre Cultura y Desarrollo, en el Banco Interamericano de Desarrollo. Washington.

Habermas, J. (2003). Teoría de la acción comunicativa. Grupo Santillana de Ediciones, S. A. Distribuidora y Editora Aguilar, Altea, Taurus, Alfaguara, S.A

Hart, A. (2001). Cultura para el desarrollo. El desafío del siglo XXI. Editorial de Ciencias Sociales. La Habana. Cuba.

Horrutinier, SP. (2007). La universidad cubana en la época actual. En: Universalización y cultura científica para el desarrollo local. La Habana, Félix Varela.

Lenin, VI. (1975). Materialismo y empiriocriticismo. Ed. Política. Cuba.

Mafessoli, M. (2001). El Tiempo de Las Tribus. Icaria, Barcelona.

Malinowski, B. (1985). Una teoría científica de la cultura. Edit. Sudamericana. Buenos Aires 
Martinell, A. (2001). Las interacciones en la profesionalización en gestión cultural. Cátedra Unesco

Maturana, H. \& Varela, F. (2002). El árbol del conocimiento, Ed. Universitaria, Santiago. Cuba

Mclaren, P. (2000). Identidad y poder. Los educadores frente al multiculturalismo. Paidós, Buenos Aires.

Montoya, RJ. (2005). La contextualización de la cultura en los currículos de las carreras pedagógicas [Tesis Doctoral En Ciencias Pedagógicas]. Cuba: Universidad de Oriente.

Morin, E. (2001). Los siete saberes necesarios para la educación del futuro. Librería El Correo de la Unesco, S.A. México.

Olmos, H. (2002). "Capacitar en Cultura: Necesidad Estratégica." Revista Pensar Iberoamérica.

Ortiz, F. (1962). Contrapunteo cubano del tabaco y el azúcar. Consejo de Cultura. La Habana. Cuba.

Ponce, D. (2011). La transposición sociocultural. Tesis doctoral. Universidad de Oriente. Cuba

Zilberstein, TJ. (2002). Reflexiones acerca de la necesidad de establecer principios para el proceso de enseñanza aprendizaje. Retrospectiva desde la didáctica Cubana. Habana. 